\title{
Construction of Two-Axis Goniophotometer for Measurement of Spatial Distribution of a Light Source and Calculation of Luminous Flux
}

\author{
F. SAMETOGLU* \\ National Metrology Institute of Turkey (TUBITAK UME), TUBITAK Gebze Yerleskesi \\ 41470, Gebze, Kocaeli, Turkey
}

(Received August 23, 2010)

\begin{abstract}
A two-axis goniophotometer is constructed at National Metrology Institute of Turkey (TUBITAK UME) for characterizing photometric properties of compact light sources. With the new device, total luminous flux emitted by a light source at both cap-up or cap-down mounting positions is calculated from the integration of the illuminance values $E(\theta, \varphi)$ measured on the goniometric spherical surface. The internal calibration of the system is performed by means of two calibrated light sources with known luminous flux values. The constructed system is also capable of measuring the luminous intensity distribution values $I(\theta, \varphi)$ of compact light sources. The uncertainty analyses of the luminous flux measurements as well as the internal calibration of the instrument are also presented herein.
\end{abstract}

PACS: 42.15.Eq, 07.60.-j, 06.20.F-, 07.60.Dq

\section{Introduction}

The luminous flux, which is a photometric quantity that describes the total optical flux of a light source as seen by a human eye, is one of the most important characteristics of a light source designed for lighting purposes [1]. The standard unit of luminous flux is lumen, which is defined as the luminous flux emitted into unit solid angle by an isotropic point source having a luminous intensity of one candela. The luminous flux is fundamentally measured by determining either the spatial integral of $I(\theta, \varphi)$ or $E(\theta, \varphi)$ on a spherical surface around the light source $[2,3]$. The maximum value in the center as well as the spatial distribution give the relevant information for light sources. In order to carry out these measurements, a positioning system is required that is capable of shifting a photometer head, on the surface of a sphere in whose center is the source of light to be evaluated. Such positioning system and photometer as a whole are termed as a goniophotometer [4].

A goniophotometer usually consists of a mechanical device for the support and positioning of the light source (lamp or luminaire) and the photometer head, together with associated device for acquiring and processing data [2]. It is required that a light source operates at its working condition (burning position at cap-up or cap-down) to measure its photometric characteristics.

* e-mail: ferhat.sametoglu@ume.tubitak.gov.tr
Light sources are very sensitive to external mechanical effects in their burning conditions during measurements, which generate an error due to vibrations and air movements [1].

Basically, three types of goniophotometers can be used to characterize light sources, which can be designed in a variety of configurations:

i) a goniophotometer which rotates the light source around two mutually perpendicular axes whose intersection is the photometric center of the goniophotometer. The photometer head is fixed. If the spatial distribution $I(\theta, \varphi)$ is of highest interest, then this simple construction might be useful. Most of the light sources have strong restrictions in the allowed burning positions, so this simple goniophotometer is only helpful in a few cases [5]. An additional error may also be caused from the shadow of their lamp-rotating mechanisms, which restricts the type of lamp for flux standards.

ii) a goniophotometer which rotates the light source around one axis only, the second rotation being given by a relative movement between the light source and the photometer head around a second axis at right angle to the first and crossing it at the photometric center of the goniophotometer. This configuration may also cause the errors stated in item (i). 
iii) a goniophotometer in which the light source does not move at all. The photometer head rotates around two mutually perpendicular axes whose crossing is the photometric center of the goniophotometer. In this case, the surface of an imaginary sphere around the light source is scanned with the photometer head.

The first type of the goniophotometer is used only for industrial purposes, mainly to measure the angular distribution $I(\theta, \varphi)$ of lamps. Remaining two are used for precise total luminous flux measurements of lamps as needed for the applications of metrology and standardized laboratories.

In order to obtain the correct photometric characteristics of the light source, the photometer head should be at a distance of at least fifteen times greater than the largest dimension of the light source being tested, so that the inverse square law can apply with reasonable accuracy [4]. Floodlights, projectors, automobile headlights and other concentrating beams should be tested at much greater distances than those of indoor luminaires. For this purposes a mirror-type goniophotometer, in which one or two mirrors are located for sending the light to the photometer head is the best solution for defeating the restrictions arising from too large distances necessary to make measurements suited in the measurement conditions specified above in laboratory situation $[6,7]$.

The portion of the luminous intensity of a light source $I(\omega)$ is better defined by subdividing the total solid angle ( $\left.\Omega=\int \mathrm{d} \omega=4 \pi\right)$ into fractional solid angles, which can only be made by using a goniophotometer. Thus, the total luminous flux $\Phi_{\mathrm{v}}$ is calculated from $\Phi_{\mathrm{v}}=\int I(\omega) \mathrm{d} \omega$.

Let us consider a sphere enclosing the light source, which is at its centre (Fig. 1) [8].

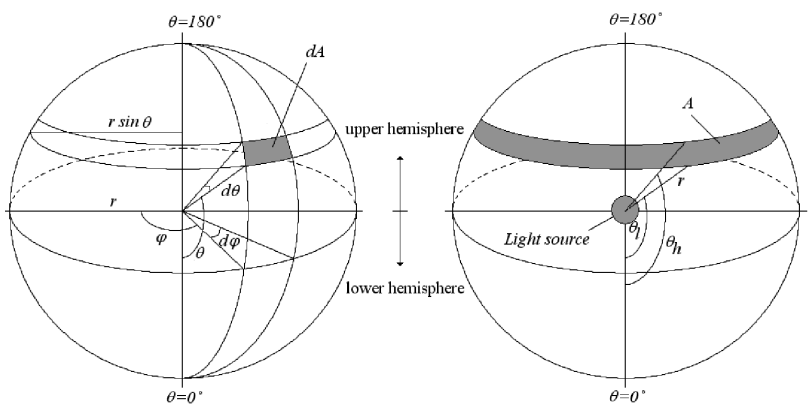

Fig. 1. Spherical surface scanned by a goniophotometer.

Let $\theta$ and $\varphi$ be the polar and the azimuthal angles in a spherical coordinate system the origin of which is at the lamp location. The solid angle is equal to the area of the zone $A$, divided by the square of the radius $r$. Thus, for an elementary zone at an angle of $\theta$ subtending an angle of $\mathrm{d} \theta$ the solid angle is

$$
\mathrm{d} \omega=\frac{\mathrm{d} A}{r^{2}}=\frac{2 \pi r \sin \theta r \mathrm{~d} \theta}{r^{2}}=2 \pi \sin \theta \mathrm{d} \theta .
$$

For the zone extending from $\theta_{\mathrm{l}}$ to $\theta_{\mathrm{h}}$

$$
\omega=2 \pi \int_{\theta_{\mathrm{l}}}^{\theta_{\mathrm{h}}} \sin \theta \mathrm{d} \theta=2 \pi\left(\cos \theta_{\mathrm{l}}-\cos \theta_{\mathrm{h}}\right) .
$$

The value is called the zone factor and for convenience it is usual to divide the sphere into zones subtending equal angles of elevation $\theta$ at the centre of the sphere. The spherical surface is divided into $n$ meridians and $m$ parallels and the luminous intensities $I_{m, n}(\theta, \varphi)$ are measured at the cross points between parallels and meridians. To find the luminous flux in a zone the zone factor is multiplied by the average $I_{m, n}(\theta, \varphi)$ in the directions of the zone. Thus, the total luminous flux emitted by the light source is calculated by

$$
\Phi_{\mathrm{v}}=\int_{\theta=0}^{\pi} \int_{\varphi=0}^{2 \pi} I_{m, n}(\theta, \varphi) \sin \varphi \mathrm{d} \varphi \mathrm{d} \theta .
$$

The derivation of the total luminous flux from illuminance $E(\theta, \varphi)$ is well suited to the realization of the standards of luminous flux. In this approach, the total luminous flux is calculated by $\Phi_{\mathrm{v}}=\int E(A) \mathrm{d} A$, where $\mathrm{d} A$ is the element of area that is illuminated by the light source at a photometric distance of $r$ (Fig. 1). So, $\mathrm{d} A=\mathrm{d} \omega \times r^{2}=2 \pi r^{2} \sin \theta \mathrm{d} \theta \mathrm{d} \varphi$. The same zonal factor method is used for finding the total luminous flux emitted by the light source here. Many national standard laboratories use this technique [9-14], where the total luminous flux is calculated by

$$
\Phi_{\mathrm{v}}=r^{2} \int_{\theta=0}^{\pi} \int_{\varphi=0}^{2 \pi} E_{m, n}(\theta, \varphi) \sin \varphi \mathrm{d} \varphi \mathrm{d} \theta .
$$

In practice, the integral of (4) is replaced by the following sum, considering (2):

$$
\Phi_{\mathrm{v}}=2 \pi r^{2} \sum_{\theta=0}^{\pi}\left[\sum_{\varphi=0}^{2 \pi} E_{m, n}(\theta, \varphi)\left[\cos \theta_{\mathrm{l}}-\cos \theta_{\mathrm{h}}\right]\right] .
$$

There are three basic requirements which must be taken into account for both measurements, $I_{m, n}(\theta, \varphi)$ and $E_{m, n}(\theta, \varphi)$. The first requirement is that the whole of the sensitive surface of the photometer head should be illuminated by the light from the whole of the light source. Thus the photometer head should have a field-of-view beyond that required [1]. The second requirement is that the light source should be exactly at the centre of the goniophotometer and the photometric distance must not be changed at any of measurement point. If the radius changes, $I_{m, n}(\theta, \varphi, r)$ and $E_{m, n}(\theta, \varphi, r)$ must be taken into account in (3) and (4) in places of $I_{m, n}(\theta, \varphi)$ and $E_{m, n}(\theta, \varphi)$, respectively $[4,11]$. The third requirement is that measurements must be carried out in a darkened room $[2,4]$. The photometer head responds to whole light coming from circumference of the lamp together with undesired reflected light (stray light), so that parts of the apparatus and the walls should be blackened. Besides in 
order to avoid heating effect on the photometric measurements it is not required to cover the goniophotometer.

\section{Instrument design}

A two-axis goniophotometer, in which the light source is kept stationary at the centre of the goniometer and the photometer head is turned around the light source, is constructed at TUBITAK UME for measuring the detector-based illuminance distribution of a compact light source and calculating luminous flux of the lumen. Two different light source holders are designed for keeping light sources to be measured at their burning conditions. Figure 2a and $\mathrm{b}$ present photographs of designed goniophotometer that measure light sources at cap-down and cap-up positions, respectively.
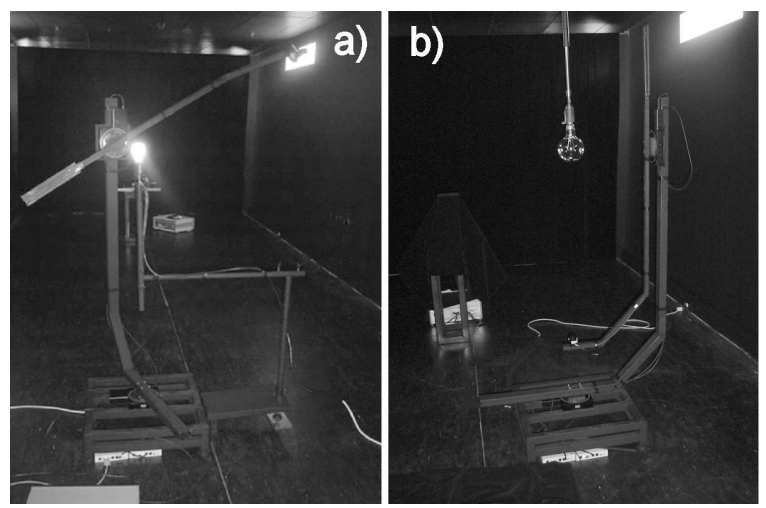

Fig. 2. Goniophotometer constructed to integrate $E(\theta, \varphi)$ : (a) the light source is operated at cap-down position, (b) the light source is operated at cap-up position.

The goniophotometer system is operated in the laboratory, environmental conditions of which are under control, the walls and the ceiling of which are finished in matt black paint. The basic components of the goniophotometer consist of a goniometer having two dc servo-motor controlled arms, height and angular adjustable light source holders, and the photometer head having a stray light reduction type. A visual programming language of National Instruments $\left(\right.$ LabVIEW $^{\circledR}$ ) is used to develop a software for controlling the instrument and acquiring data [15].

\subsection{Mechanical design of the goniometer}

The goniometer consists of a mounting-base, a computer-controlled outer arm coupled to the mounting-base and a computer-controlled interior arm coupled to the outer arm. An overall view of the goniometer is given in Fig. 3, which also shows its main components.

The mounting-base has square-shaped construction $(605.0 \mathrm{~mm}(W) \times 605.0 \mathrm{~mm}(L) \times 170.0 \mathrm{~mm}(H))$ and made from the black-anodized hollow metal profile $(40.0 \mathrm{~mm}(W) \times 40.0 \mathrm{~mm}(H))$ having thickness of $1.0 \mathrm{~mm}$. The mounting-base is equipped with four

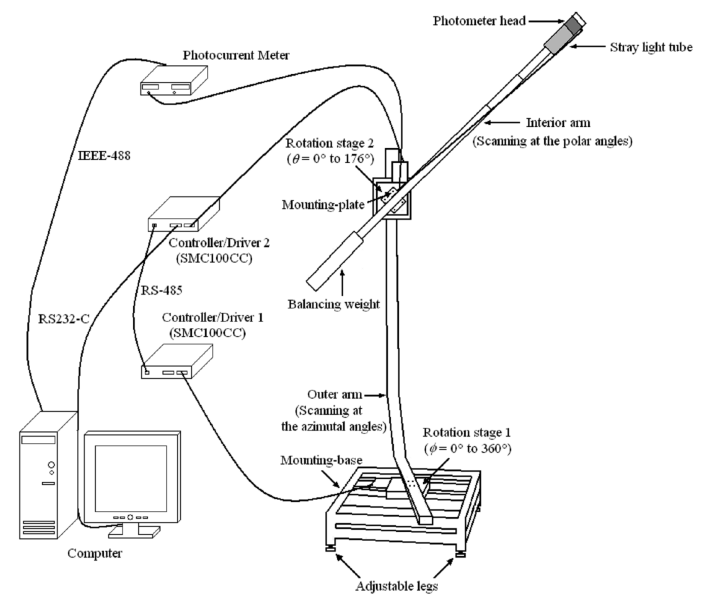

Fig. 3. An overall view of the goniometer.

adjustable legs, which are used for keeping the goniometer in balance and to operate it in vibration-free condition. A motorizing rotation stage 1 (RS1) having precision homing and accurate positioning, manufactured by Newport Corporation (URS150BCC), is used to move the $L$-shaped outer goniometer arm in azimuthal angles $(\varphi)$ from $0^{\circ}$ to $360^{\circ}$. This arm is manufactured of black-anodized hollow metal profile $(60.0 \mathrm{~mm}(W) \times$ $40.0 \mathrm{~mm}(H)$ ) having thickness of $1.2 \mathrm{~mm}$. A square mounting-plate $(90.0 \mathrm{~mm} \times 90.0 \mathrm{~mm})$ having thickness of $3.0 \mathrm{~mm}$ is mounted to the bottom centre of the arm for assembling the outer arm to the centre of the RS1. The second square mounting-plate $(190.0 \mathrm{~mm} \times 190.0 \mathrm{~mm})$ having thickness of $3.0 \mathrm{~mm}$ is screwed to the upper part of the outer arm so that to assemble the second rotation stage of URS150BCC (RS2). This rotation stage acts the interior arm of the goniometer within the polar angles $(\theta)$ from $0^{\circ}$ to $176^{\circ}$. The interior arm, which has configuration of $L$-shape and used for assembling the photometer head and scanning of the test lamp within the polar angles, is manufactured of standard black-anodized hollow metal profile $(30.0 \mathrm{~mm}(W) \times 20.0 \mathrm{~mm}(H))$ having thickness of $1.0 \mathrm{~mm}$. A square mounting-plate $(90.0 \mathrm{~mm} \times 90.0 \mathrm{~mm})$ having thickness of $3.0 \mathrm{~mm}$ is mounted to the rear side of the arm so that to fix the centre of the interior arm to the centre of the RS2. An appropriate balancing weight is also added to a shorter side of the interior arm for obtaining balanced and un-friction movement in whole rotation.

Operation of RS1 and RS2 is performed by means of single-axis motor controllers, SMC100CC's [16], manufactured by Newport Corporation. The manufacturer stated that the absolute uncertainty in the positioning of each motor is $0.023^{\circ}$, which contributes on uncertainties of $0.07 \%$ in both $\Delta \varphi$ and $\Delta \theta$ steps. Both controllers are connected to each other through the internal RS-485 communication link and transferring data between a computer and the first controller is carried out via a serial communication of the RS-232 interface. Input 
parameters of both motorized stages, such as acceleration/deceleration $\left(10^{-6}-10^{12}\right)$ and velocity $\left(0.01-80^{\circ} / \mathrm{s}\right)$, can be adjusted before use so that to increase or decrease the total measuring time from some tens of minutes to as long as a few hours.

The photometer head to be used for detection on goniphotometer is composed of a silicon photodiode, a photopic filter and an aperture combination. It is mounted to nearly the extremity point of the interior arm, so that the centres of the photometer head aperture and the filament of the light source are to be at the same optical axis. All rotational axes intersect at centre of the lamp centre, so that measurements at all angles are centred on the same sphere surface. The radius of the sphere described by the arm of the photometer is aligned to $r=1.32 \mathrm{~m}$.

\subsection{Lamp holders}

Two different types of lamp holders are designed to keep light sources at their arbitrary burning positions (Fig. 4a and b) and to measure $E(\theta, \varphi)$ or $I(\theta, \varphi)$.

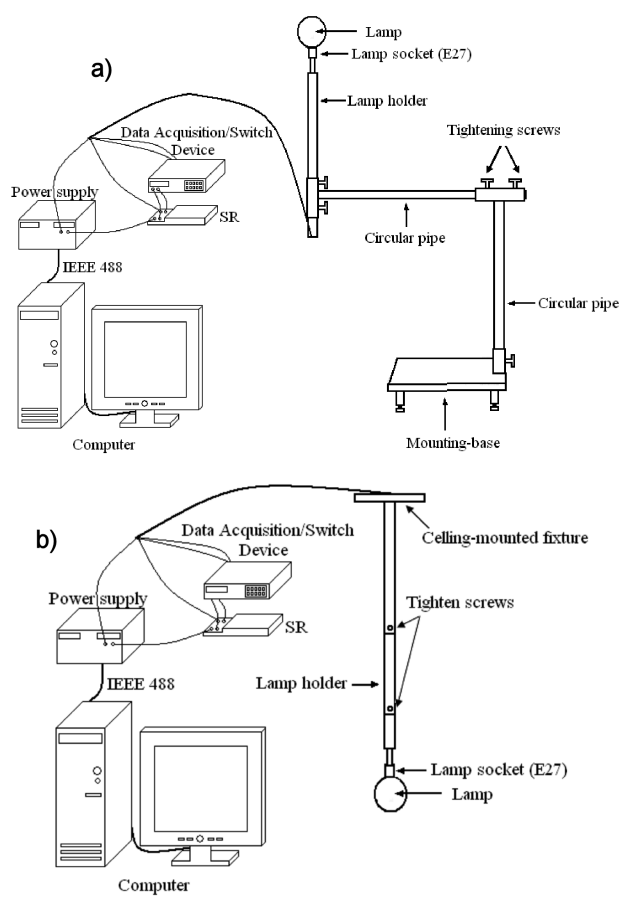

Fig. 4. Lamp holders designed for keeping light sources: (a) at cap-down position, (b) at cap-up position.

Both lamp holders have height-adjustable mounting fixture and special designed lamp sockets, each of which has an E27 screw-in base with a four-pole configuration providing separate operating current and base voltage sensing wires [17]. The lamp voltage is measured directly between two contacts whereas the lamp current is measured as the voltage across a calibrated standard shunt resistor (SR). 20-channel Agilent 34970A Data Acquisition/Switch device is used to measure electrical parameters (current and voltage) of tested light sources.

\subsection{Description of the designed photometer head}

Internal components of the thermally-stabilized photometer head are depicted in Fig. 5a. The photometer head consists of a Hamamatsu 1227-1010BQ photodiode with an active area of $10 \mathrm{~mm}^{2}$, a PRC manufactured $V(\lambda)$-correction filter with a diameter of $15 \mathrm{~mm}$ and an aperture with an area of $0.05 \mathrm{~mm}^{2}$, which are assembled in the cylindrical housing.

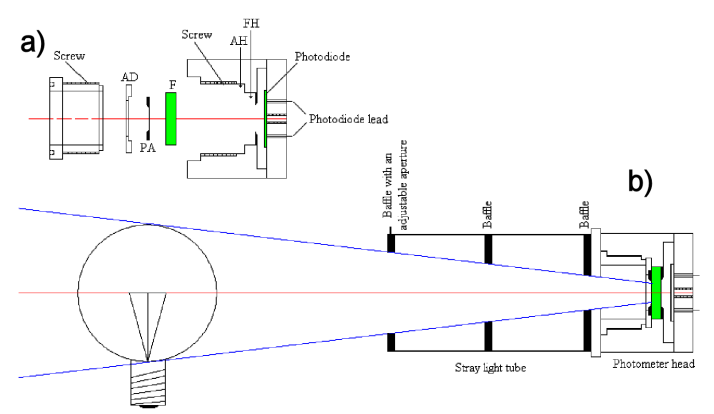

Fig. 5. The photometer head designed for measuring $E(\theta, \varphi)$ or $I(\theta, \varphi)$ : (a) components, (b) assembled to the stray light tube. $\mathrm{AD}$ - aperture disc, $\mathrm{PA}$ - precision aperture, $\mathrm{F}-V(\lambda)$-filter, $\mathrm{AH}-$ aperture holder, $\mathrm{FH}$ - filter holder.

Luminous responsivity of a silicon photodiode has been found to be independent of the orientation of the goniophotometer arm [9]. The overall distance between the photodiode surface and the entrance aperture of the photometer head is $15 \mathrm{~mm}$. The $V(\lambda)$-filter is glued to a holder and incorporated immediately in front of the photodiode to make the relative spectral responsivity of the assembly close to the CIE- $V(\lambda)$ function. The aperture is glued on a designed aperture disc and the disc is placed to an aperture holder. The generated photocurrent at the output of the photometer head is measured via a calibrated $4 \frac{1}{2}$-digit photocurrent-meter (I $1000 \mathrm{SD}$ ) manufactured by Lichtmesstechnik GmbH (LMT), which is connected to computer via a GPIB interface.

The spectral responsivity, uniformity and linearity of the photometer head as well as the spectral transmittance of the $V(\lambda)$-filter were characterized by the TUBITAK UME detector calibration and characterization facilities [18-20]. The diameter of the monochromatic beam was aligned to $\approx 2 \mathrm{~mm}$ and a substitution method with the reference trap detector was used to obtain the relative spectral responsivity variation of the photometer head. Closeness of a photometer head response to the CIE$-V(\lambda)$ function is the most important criterion, which is characterized by calculating error factor of $f_{1}^{\prime}[21]$. The obtained measurement results for the spectral transmittance of the $V(\lambda)$ filter, the spectral responsivity of the photometer head and the difference between the spectral responsivity and the CIE- $V(\lambda)$ function are shown in Fig. 6.

Using spectral characteristic results of each the component of the photometer head, factor $f_{1}^{\prime}$ and the illumi- 


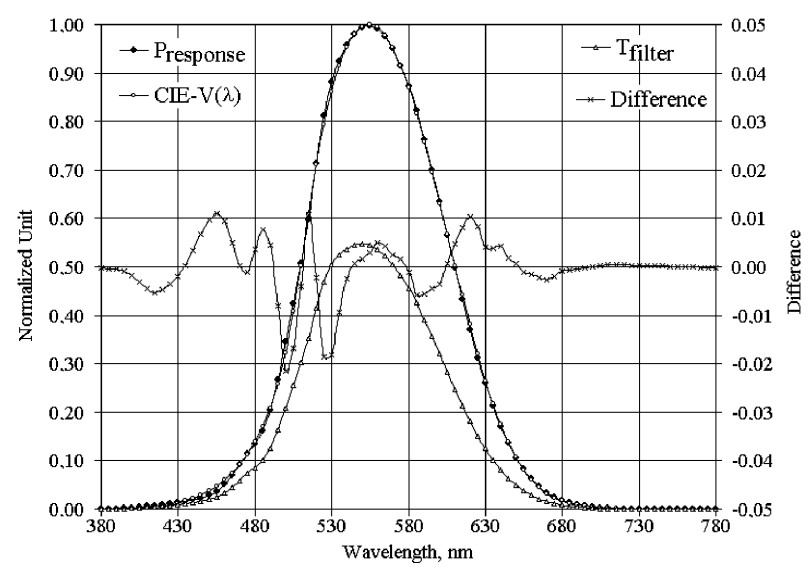

Fig. 6. The spectral responsivity of the photometer head and the difference between the CIE- $V(\lambda)$ function.

nance responsivity $s_{\mathrm{v}}$ of the photometer head were calculated as $1.4 \%$ and $11.910 \mathrm{nA} / \mathrm{lx}$, respectively.

The photometer head linearity was checked at wavelengths of $543.2 \mathrm{~nm}$ and $632.8 \mathrm{~nm}$ over an output photocurrent range of $2 \times 10^{-3}$ to $2 \times 10^{-10}$ A [20], which corresponds to be used for illuminance measurements within $8.4 \times 10^{-3}$ to $8.4 \times 10^{3} \mathrm{~lx}$. For typical working photocurrents of $30 \mathrm{nA}$ to $30 \mu \mathrm{A}$, the nonlinearity was observed to be $\pm 0.02 \%$. The uniformity of the photometer head was measured by using a computer-controlled optical setup, where the acceptance area of the photometer head was scanned over the central area of $10 \mathrm{~mm} \times 10 \mathrm{~mm}$ at wavelength of $632.8 \mathrm{~nm}$ [20]. The responsivity of the photometer head was recorded for each scanning interval of $10 \mu \mathrm{m}$ in entrance aperture of the photometer head. The uniformity was finally obtained by dividing these responses at the selected points to the maximum responsivity value. It was found that the uniformity within the $6 \mathrm{~mm}$ diameter is $\approx 0.1 \%$ with a relative standard uncertainty of $0.01 \%$.

The distance from the entrance aperture of the photometer head to the center of the goniometer was aligned to $1.32 \mathrm{~m}$ by using a calibrated length gauge and verified at least ten different points of the goniometer [17]. At the worst case, the thickness of the gauge mark line, $\Delta d=0.4 \mathrm{~mm}$, showing the distance on the gauge was used as the distance setting uncertainty, which corresponds to the photometric uncertainty of $0.06 \%$.

The exclusion of extraneous light, which is known as the stray light, is achieved by the use of a baffled stray light reduction tube with length of $150 \mathrm{~mm}$ and diameter of $50 \mathrm{~mm}$ (Fig. 5b). The designed tube has two invariable mechanical baffles and a baffle having an adjustable aperture, whose functions are to shield the light coming from sources outside the field-of-view of the photometer head. The tube is attached in the front of the photometer head housing. The inside walls and components of the tube are finished in matt black so that to reduce internal reflections.

\subsection{Control software}

Except for luminous flux, the determination of $E(\theta, \varphi)$ or $I(\theta, \varphi)$ of light source in space involves the use of a coordinate system to define the direction of the illumination. Many light sources, particularly those with small incandescent sources, have an axis of symmetry. The polar curve is the most common method of presenting both the distributions for those types of light sources. However for light sources with concentrating beams, such as spotlights emitting all their luminous flux within a few degrees of solid angle, Cartesian coordinates are preferred because of the need for more precision than a polar curve allows [1]. After calculating of the total luminous flux, $I(\theta, \varphi)$ distribution may also be expressed in candelas per 1000 lumens.

A software program, written within the National Instruments LabVIEW ${ }^{\circledR}$ graphical programming compiler [15], is developed to automate the instrument and data acquisition for measuring $E(\theta, \varphi)$ or $I(\theta, \varphi)$ and determining the luminous flux emitted by light source. While other programming systems use text-based languages to create lines of code, LabVIEW ${ }^{\circledR}$ uses a graphical programming language called $\mathrm{G}$ to create programs in a pictorial form, eliminating a lot of the syntactical details. The block diagram of the constructed system is depicted in Fig. 7.

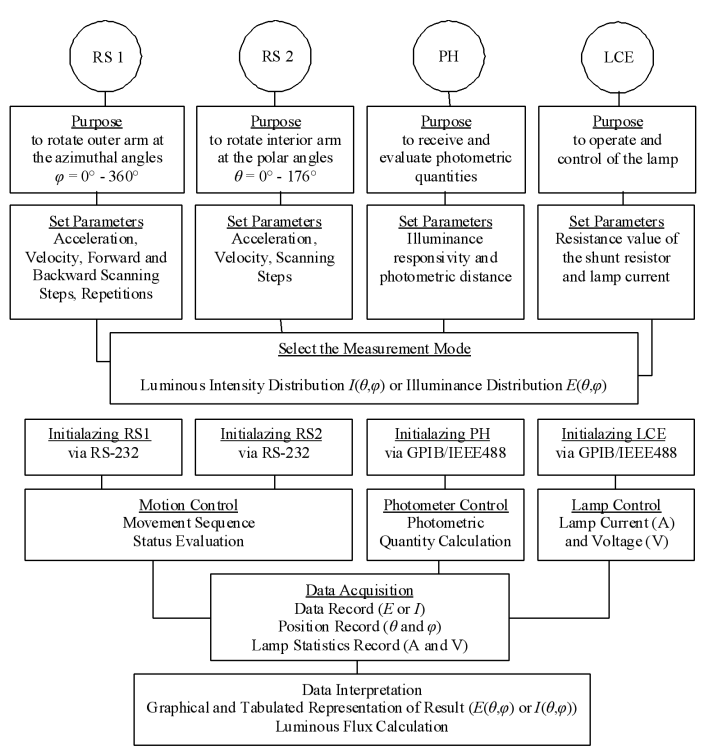

Fig. 7. Block diagram of the system. RS1 and RS2 are the first and second rotation stages, $\mathrm{PH}$ is the photometer head, LCE is the lamp control equipments.

A personal computer is used to operate the goniophotometer, to control the motion of the goniometer, to set up and to acquire data from the photometer head and to operate the lamp being tested at desired current. Figure 7 shows that computer controls the first (RS1) and the second motors (RS2), the photometer head $(\mathrm{PH})$ and electronic equipments used for operating and measuring 
light source parameters (LCE). Before starting a measurement, operator sets input parameters of each motor (acceleration, deceleration, velocity, etc.). RS1 rotates the outer arm at the azimuthal angles from $0^{\circ}$ to $360^{\circ}$ with the angular steps of $\Delta \varphi$ (clockwise scanning, CWS). Then RS2 acts the interior arm to the first step within the polar angles. After that, RS1 acts the outer arm backward from $360^{\circ}$ to $0^{\circ}$ (counterclockwise scanning, CCWS). This procedure continues automatically until the total spherical surface is scanned. Therefore scanning steps for both the azimuthal and the polar angles and repetition numbers of operations should be set before the measurements. For example, it is necessary to scan $E(\theta, \varphi)$ of a light source with $10^{\circ}$ steps in both the polar and the azimuthal angles. Therefore scanning step of RS2 should be set to $10^{\circ}$, the CWS and CCWS scanning steps for RS1 should sequentially be set to $10^{\circ}$ and $-10^{\circ}$ and repetition numbers of RS1 and RS2 should sequentially be set to $36^{\circ}$ and $18^{\circ}$, respectively.

After setting the above parameters, measurement mode is selected between $E(\theta, \varphi)$ and $I(\theta, \varphi)$. The front panel of the software contains also the polar graph and table selection menu to present measurement results visually and the total luminous flux result of the light source if $E(\theta, \varphi)$ condition is selected. By default, there are five types of polar diagrams: $0^{\circ} \ldots 90^{\circ}, 90^{\circ} \ldots 180^{\circ}$, $180^{\circ} \ldots 270^{\circ}$ and $270^{\circ} \ldots 360^{\circ}$ and $0^{\circ} \ldots 360^{\circ}$.

The standard program execution control structure of sequence structure, located within the structures subpalette of the floating functions palette of the LabVIEW ${ }^{\circledR}$ program, is used for developing of the software. The structure includes totally five sub-structures. The first sub-structure consists of operation commands for the position and the photocurrent data acquisition from each of the encoder and the photometer head at $(\theta, \varphi)=\left(0^{\circ}, 0^{\circ}\right)$. The second sub-structure includes operation commands for manipulating the outer arm of the goniometer in the CWS direction with a setted angular steps $(+\Delta \varphi)$ while the interior arm is kept stationary at $0^{\circ}$ and collecting photocurrents at each goniometer position. The standard "knob" control, located within the numeric subpalette of the floating controls palette, and the standard "for loop" structure are used for manipulating RS1 with the setted angular steps and defining repetition number of operation, respectively. The second "knob" control is connected to the count terminal $(N)$ of the "for loop" structure for performing repeated measurements for RS1 ( $N=36$ for scanning with $10^{\circ}$ steps). The third sub-structure consists of operation commands for manipulating the interior arm with RS2 to the first setted forward step and performing the CCWS with the setted angular steps $(-\Delta \varphi)$. The "knob" control is connected to the count terminal $(N)$ of the "for loop" structure for performing repeated measurements with the setted angular steps $\left(N=18\right.$ for scanning with $10^{\circ}$ steps $)$. The forth and the last sub-structures include operation commands for manipulating both the arms to start position (home position) and taking the photocurrent measurement at $(\theta, \varphi)=\left(0^{\circ}, 0^{\circ}\right)$ again to check the stability of the test light source during measurement.

In order to control each motor and to get angular position from each encoder, special LabVIEW ${ }^{\circledR}$ drivers developed by Newport Corporation are used such as "port init.vi", "home.vi", "move relative.vi", "wait for stop 1 axis.vi" and "tell current position.vi" and "close.vi" [16]. The "port init.vi" driver is used for initializing each motor controller to the computer via a COM port of the RS-232 communication (baud rate $=57600$, data bits $=8$, parity $=$ none, stop bits $=1$, flow control $=\mathrm{XON} / \mathrm{XOFF}$ and terminal character $=C_{\mathrm{R}} / L_{\mathrm{F}}$ ) by setting the timeout of the driver to $300 \mathrm{~ms}$. The "home.vi" driver starts the execution of the HOME search sequence of the motor as defined by the set home type command. The "move relative.vi" driver is a move command. Displacement unit of each driver was set to $0.0^{\circ}$. Waiting of each controller while it is in a moving state and checking if the controller is in ready state were accomplished by the "wait for stop_1 axis.vi" driver. The "tell current position.vi" driver is then used for recording of the encoder feedback position and finally serial port of communication is closed by using close.vi command.

A LabVIEW ${ }^{\circledR}$ based data acquisition driver is also developed for initializing the photocurrent-meter (I 1000 $\mathrm{SD})$ to a computer via a GPIB communication through address string of 16 and to take off readout from the photometer head. The manufacturer of the photocurrent-meter, Lichtmesstechnik $\mathrm{GmbH}$, stated that the data transmission from the instrument is terminated by a $L_{\mathrm{F}}$ (line feed) terminator and at the end of the transmission normally no EOI-signal should be sent. The standard instrument I/O functions such as "GPIB Write" and "GPIB Read" are used separately within individual sub-structures for controlling the photocurrent-meter. The data string of the "GPIB Write" is set to "enter", timeout to 1000 and terminating of the "GPIB Write" mode to 5 (append $L_{\mathrm{F}}$ to the string but do not send EOI). The byte count of the "GPIB Read" is setted to 15 according to instruction given by the manufacturer. Then the data terminal of the "GPIB Read" is connected to the string input of the "Rotate String" function. The standard LabVIEW ${ }^{\circledR}$ functions of "Replace Substring", "Reverse String", "Replace Substring", "Reverse String" and "Fract/Exp String to Number" are then connected to each other for obtaining numerical value from the photocurrent-meter.

The obtained photocurrent value $(y)$ at the output of the photometer head is divided by the illuminance responsivity value of the photometer head $\left(s_{\mathrm{v}}\right)$ for obtaining $E(\theta, \varphi)$ at each angular position $\left(E=y / s_{\mathrm{v}}\right)$. The measurement results are automatically transferred to a table and polar graph that are created by using standard LabVIEW ${ }^{\circledR}$ commands. $I(\theta, \varphi)$ is calculated from multiplying the obtained illuminance value by square of the photometric distance $\left(r^{2}\right)$. The software can also present graphical presentation of $I(\theta, \varphi)$ distribution expressed in candelas per 1000 lumens. 


\section{Experimental results}

$E(\theta, \varphi)$ distribution measurements were performed by using a set of three previously calibrated luminous flux standards manufactured by Philips company, P12, P14, $\mathrm{P} 15$, and the total luminous flux of each light source was calculated according to (5). All light sources are incandescent type with a nominal power of $24 \mathrm{~V} / 100 \mathrm{~W}$ and have an opal bulb with inside frosted feature. The dimensions (diameter and height) of the lamp bulb are $60.0 \mathrm{~mm} \times 75.0 \mathrm{~mm}$.

Each of lamps was sequentially mounted on a vertical pipe at cap-up position (Fig. 4b) and adjusted so that the filament centre was at the centre of the goniophotometer. This alignment was verified through two small lasers that emit their beams at the centre of the goniophotometer. The reference point, on which the azimuthal and the polar angles are zero, was settled exactly below the lamp (at the nadir position). A DC power supply (PTN 125-20 series), manufactured by Heinzinger Electronics $\mathrm{GmbH}$, was used to operate the lamp in constant-current mode and at a fixed polarity. The power supply was connected to the computer via a IEEE-488 interface. When the lamp current stabilization was reached to levels of $10^{-5} \mathrm{~A}$, the goniophotometric measurements started.

Angular steps in both the azimuthal and the polar angles were set to $+\Delta \varphi=-\Delta \varphi=\Delta \theta=10^{\circ}$ for sampling data at $10^{\circ}$ steps. The goniophotometer performs "stop-go" mode of operation. Thus, the acceleration and deceleration parameters of the goniometer motors were setted to $0.1 \mathrm{~m} / \mathrm{s}^{2}$ and motion speeds in the polar and the azimuthal angles to $5^{\circ} / \mathrm{s}$ and $10^{\circ} / \mathrm{s}$, respectively.

The measurements were started to measure $E_{m, n}(\theta, \varphi)$ at the reference position, by taking the first measurement of $E_{m, n}(\theta, \varphi)=E_{m, n}\left(0^{\circ}, 0^{\circ}\right)$. Then $E_{m, n}(\theta, \varphi)$ was determined by rotating the photometer head $360^{\circ}$ on its own axis. This process is performed so as to check whether or not the alignment of the photometer head along with the goniophotometer arms is exact. After the alignment check process, the photometer head was increased as the started angular step size $\Delta \theta$, at the polar angle and it stopped. Then the goniophotometer moved the photometer head in the azimuthal angles from $0^{\circ}$ to $360^{\circ}$ by means of the CWS with angular steps of $\Delta \varphi$. Once the photometer attained $\varphi=360^{\circ}$, the goniophotometer took the photometer head forward at polar axis with the angular step of $\Delta \theta$ and it waited for the completion of the azimuthal CCWS stop, to be acted from $\varphi=360^{\circ}$ to $0^{\circ}$ with angular steps of $\Delta \varphi$. This process, as a result, was maintained with CWS and CCWS at the azimuthal angles as long as $\Delta \theta$ reached to $176^{\circ}$. The photometer head was brought to its home position after all the measurements were completed and measurements were repeated at the reference point again. The photometer head was stopped for $1 \mathrm{~s}$ during each angle-step to collect the photocurrent generated by the photometer head and mean $E_{m, n}(\theta, \varphi)$ value was calculated. $E_{m, n}(\theta, \varphi)$ of tested lamp was totally measured in 667 points around the lamp. The collected data were then used to calculate the total luminous flux of the lamp.

Figure 8a shows polar distribution graph of $E_{m, n}(\theta, \varphi)$ for one of measured lamps at azimuthal angle of $\theta=90^{\circ}$.

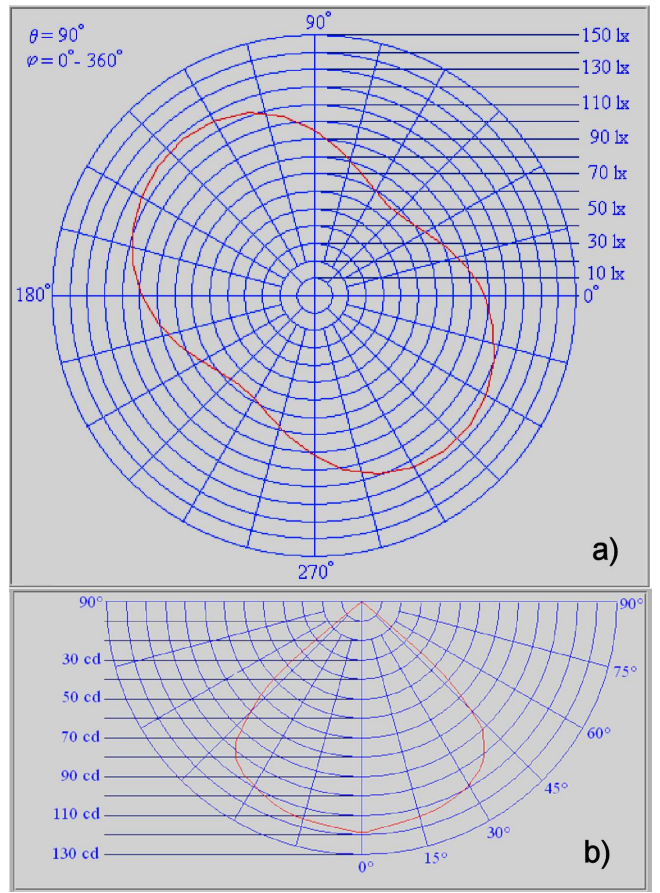

Fig. 8. Polar distribution graphs: (a) polar chart measured for $E_{m, n}(\theta, \varphi)$ at the azimuthal angle of $\theta=90^{\circ}$; (b) polar chart measured for $I_{m, n}(\theta, \varphi)$.

The lamp has a nearly homogeneous distribution and emits all its light within near-elliptical shape. The maximum illuminance was observed at the order of $120 \mathrm{~lx}$ from the angles of $\varphi=120^{\circ}-150^{\circ}$ and at the opposite side from $\varphi=300^{\circ}-330^{\circ}$. At the other hand, the lamp produced pure illuminance of $\approx 50 \mathrm{~lx}$ from azimuthal angles of $\varphi=30^{\circ}-60^{\circ}$ and $\varphi=210^{\circ}-240^{\circ}$. The reason of such variation may be described by the geometric structure of the filament.

Table I reports the total luminous flux results obtained by using the collected $E_{m, n}(\theta, \varphi)$ values and (5) as well as the internal comparison results of the luminous flux values.

TABLE I

Differences between the certificated and measured luminous flux values of three lamps.

\begin{tabular}{c|c|c|c|c}
\hline \hline Lamps & $\begin{array}{c}\text { Measured } \\
{[\mathrm{lm}]}\end{array}$ & $\begin{array}{c}\text { Certification } \\
{[\mathrm{lm}]}\end{array}$ & $\begin{array}{c}\text { Difference } \\
{[\%]}\end{array}$ & Ratio \\
\hline P12 & 868.4 & 865.0 & -0.39 & 1.0039 \\
P14 & 1183.5 & 1185 & 0.13 & 0.9987 \\
P15 & 1313.2 & 1319 & 0.44 & 0.9956
\end{tabular}

The second column refers to the obtained values in lumens, whereas the third column shows the luminous flux 
values of lamps taken from their individual certificates. The third and the last columns show the difference between the measured and the certificate values in percents and the ratio between two results. By taking the ratio results into account, it seems that the mean value is 0.9994 with a standard deviation of \pm 0.0042 . Consequently, the total luminous flux values agreed within $0.45 \%$.

The goniophotometer was also used to measure $I(\theta, \varphi)$ of an automotive headlighting lamp (12 V/55 W Philips H7). The lamp was kept at cap-down position (Fig. 4a) and operated at constant-current mode. The same scanning intervals $\left(+\Delta \varphi=-\Delta \varphi=\Delta \theta=10^{\circ}\right)$ and data collecting technique were chosen and used. $I(\theta, \varphi)$ at each position of the photometer head was obtained from multiplying the measured illuminance by the square of the photometric distance. Figure $8 \mathrm{~b}$ presents $I(\theta, \varphi)$ graph for tested lamp. The direction of greatest intensity is directly below the center of the bulb $(I(\theta, \varphi)=120 \mathrm{~cd})$. The lamp emits all its light downwards in a cone subtending about $90^{\circ}$.

\section{Measurement uncertainty}

Uncertainty of the total luminous flux measurements by using the goniophotometer was analyzed and given as an uncertainty budget in Table II. All known uncertainty components are included, whether significant or not.

TABLE II

The uncertainty budget for calculating of the total luminous flux by means of the goniophotometer.

\begin{tabular}{|c|c|}
\hline Source of uncertainty & $\begin{array}{c}\text { Relative standard } \\
\text { uncertainty [\%] }\end{array}$ \\
\hline \multicolumn{2}{|l|}{ photometer head } \\
\hline illuminance responsivity & 0.15 \\
\hline non-linearity & 0.02 \\
\hline spatial non-uniformity & 0.01 \\
\hline IR-leakage & 0.02 \\
\hline drift & 0.03 \\
\hline \multicolumn{2}{|l|}{ goniometer } \\
\hline polar scanning $\left(0^{\circ}<\theta<176^{\circ}\right)$ & 0.07 \\
\hline azimuthal scanning $\left(0^{\circ}<\varphi<360^{\circ}\right)$ & 0.07 \\
\hline photometer perpendicularity alignment & 0.03 \\
\hline verticality alignment of lamp shaft & 0.03 \\
\hline lamp-to-photometer distance & 0.06 \\
\hline \multicolumn{2}{|l|}{ measurement } \\
\hline lamp current stability & 0.01 \\
\hline stray light & 0.08 \\
\hline photocurrent & 0.02 \\
\hline reproducibility & 0.13 \\
\hline combined uncertainty $(k=1)$ & 0.25 \\
\hline expanded uncertainty $(k=2)$ & 0.50 \\
\hline
\end{tabular}

The illuminance responsivity of the photometer head has been calibrated at TUBITAK UME using the new detector-based illuminance scale with an expanded uncertainty of $0.30 \%(k=2)[20]$. The photometer head is recalibrated annually by comparing it with the absolutely calibrated filter-radiometers constructed for the realization of the candela. The drift of the illuminance responsivity value since its last calibration was estimated from its calibration history to be $-0.009 \mathrm{nA} / \mathrm{lx}(\approx 0.08 \%)$ with a standard deviation of 0.0042 . Thus, the standard uncertainty of the drift value was estimated with a rectangular type of distribution and found to be $0.03 \%$.

The lamp current is monitored via the voltage drop across a calibrated dc current shunt $(0.1 \Omega)$. The voltage drop during the measurement was obtained from the arithmetic mean of 20 readings as 0.379981 V. Each reading was obtained by subtracting the offset voltage from the reading. Thus, the standard uncertainty of the lamp current stability is obtained as $0.01 \%$, which is the result of relative standard deviation of repeated measurements.

The stray light effect, which is known also as unwanted light, is more problematic when a detector-based method was used. The stray light is commonly manifested as random scatter or directional reflection of the light from surrounding. This effect was measured as follows. A screen with a circular beveled aperture (a little bit more than field-of-view of the photometer head) was used at the front surface of the photometer head to mask stray rays. Then the amount of stray light was calculated by taking mean ratio of the photometer head signals at lamp off and lamp on conditions. The measurements were repeated at eight mutual points and the standard deviation of results was used as an uncertainty in the stray light measurements $(0.08 \%)$.

The photocurrent generated at the output of the photometer head is measured with a LMT I-1000 SD type of the digital photocurrent-meter. The measured photocurrent at each position was taken from the average of six repeated readings and the uncertainty of the photocurrent measurements was estimated as $0.02 \%$, which is the standard deviation of results.

The total luminous flux measurements for each lamp were repeated six times by removing and remounting the same lamp again. The uncertainty in the reproducibility was therefore obtained from the maximum standard deviation result.

\section{Conclusion}

At TUBITAK UME, a computer-controlled two-axis goniophotometer was constructed to calculate the total luminous flux by measuring and integrating $E(\theta, \varphi)$ values of modern light sources such as LEDs, compact incandescent lamp, halogen lamps or compact fluorescent lamps. Apart from the mechanical adjustment, every part of the goniophotometer is computer-controlled. A slip-ring assembly that is generally used for transferring the electrical wires of the motors and the lamp are not used in the designed configuration, which could cause unwanted current fluctuations. In the designed configuration, the tested light source is kept stationary at the centre of the goniometer that acts by using two 
dc servomotor-based rotation stages and the surface of an imaginary sphere around the light source is scanned with the calibrated photometer head. The scanning principle is briefly defined that when the photometer head is moved on polar angles from $0^{\circ}$ to $176^{\circ}$ during the scanning process, the azimuthal angles are scanned as CWS and CCWS rotations of the photometer head for each polar angle step.

The expanded uncertainty in the total luminous flux calculation by means of the constructed goniophotometer was analysed and estimated as $0.5 \%(k=2)$. The estimated uncertainty value is valid also for the luminous intensity distribution measurements. The analysis results and formed uncertainty budget showed that estimated uncertainty is more influenced by the uncertainties arised from the illuminance responsivity of the photometer head $(0.15 \%, k=1)$ and reproducibility of results $(0.13 \%, k=1)$.

The constructed measurement system is used to measure the total luminous flux of three luminous flux standard lamps, which have previously been calibrated by the Physikalisch-Technische Bundesanstalt (PTB) Germany. Internal comparison of results showed that the agreement between two results is within the estimated uncertainty.

The distance between the photometer head aperture and the goniometer centre is $1.32 \mathrm{~m}$, which gives an opportunity to measure photometric properties of light sources having dimensions no bigger than $132 \mathrm{~mm}$. The spectral and colorimetric properties of light sources can also be measured by replacing the photometer head by a miniaturized spectrometer.

\section{References}

[1] M.A. Cayless, A.M. Marsden, Lamps and Lighting: A Manual of Lamps and Lighting, Edward Arnold, London 1983.

[2] Commission Internationale de l'Eclairage, The Measurement of Absolute Luminous Intensity Distributions, CIE Publication 70, Vienna 1987.
[3] Commission Internationale de l'Eclairage, The Measurement of Luminous Flux, CIE Publication 84, Vienna 1987.

[4] Commission Internationale de l'Eclairage, The Photometry and Goniophotometry of Luminaires, CIE Publication 121, Vienna 1996.

[5] R.E. Levin, Lighting Des. Appl. 12, 28 (1982).

[6] V.F. Munoz-Martinez, J. Seron-Barba, R. Molina-Mesa, J.M. Gomez-de-Gabriel, J. Fernandez-Lozano, A. Garcia-Cerezo, Metrologia 43, 185 (2006).

[7] M. Shaw, T. Goodman, Appl. Opt. 47, 2637 (2008).

[8] D. Förste, Licht Forschung 1, 30 (1979).

[9] W.J. Brown, Metrologia 15, 167 (1979).

[10] Y. Zong, Y. Ohno, in: Proc. CIE 26th Session, CIE, Beijing 2007, p. 179.

[11] G. Sauter, Metrologia 32, 685 (1995).

[12] M.L. Rastello, E. Miraldi, P. Pisoni, Appl. Opt. 35, 4385 (1996)

[13] M.E. Thain, F. Hengstberger, J. Phys. E: Sci. Instrum. 15, 675 (1982).

[14] M. Lindemann, R. Maass, Mapan - J. Metrol. Soc. India 24, 143 (2009).

[15] L.K. Wells, J. Travis, LabVIEW for Everyone: Graphical Programming Made Even Easier, Bk\&CD-ROM edition, Prentice Hall, 1997.

[16] Newport Corporation. Single-Axis Motion Controller/Driver for DC or Stepper Motor: SMC100CCESMC100CC - LabView Drivers Manual (retrieved 22 July 2009).

[17] F. Sametoglu, Appl. Opt. 47, 5829 (2008).

[18] F. Samedov, O. Bazkir, European Phys. J. Appl. Phys. 30, 205 (2008).

[19] F. Sametoglu, Opt. Lasers Eng. 45, 36 (2007).

[20] F. Sametoglu, Opt. Rev. 13, 326 (2006).

[21] Commission Internationale de l'Eclairage, Characterizing Illuminance Meters and Luminance Meters, CIE Publication 69, Vienna 1987. 